\title{
Surgical Ablation of Canal Ear in a Dog with Ceruminous Adenocarcinoma Ear Tumor
}

\author{
Jacqueline MOCANU*, Andrei TĂNASE, Teodora GÂRDAN, Cerasella VASILESCU, Anca CUCOS, Bianca \\ BOFAN
}

University of Agronomic Sciences and Veterinary Medicine of Bucharest, Faculty of Veterinary Medicine of Bucharest, 060033, Splaiul Independentei Street, no.105, District 5, Bucharest, Romania

Department of Surgery, Clinical Sciences

*Corresponding author: jacqueline_mocanu@yahoo.com

Bulletin UASVM Veterinary Medicine 73(1) / 2016,

Print ISSN 1843-5270; Electronic ISSN 1843-5378

DOI:10.15835/buasvmcn-vm: 11550

\begin{abstract}
Ceruminous gland adenocarcinoma is the primary malignant tumor of the sweat glands that is found in the external ear canal. Though rare, it is one of the most common malignant tumor of the ear canal in adult and older dogs. These tumors may be pedunculated or broad based but rise above the level of the epithelium. Surgical removal of benign ear canal tumors may be accomplished via lateral ear canal resection for access to the tumor mass. No data are available on the efficacy of chemotherapy for otic tumors of dogs and cats.Prognosis of patients with otic neoplasms can best be determined by histopathologic examination of removed tissues.In this article the authors describe the case of a dog breed German Brac, female 5 years old who relapsed chronic otitis present. After otoscopic exam was a friable globular formation into the deep external ear canal.We performed a total ear canal ablation (TECA) The surgery was performed under general anesthesia inhalation. The reasons for this type of anesthesia were duration of surgery determined by the anatomical complexity of the area and the rich innervation of the area. Postoperatively we generally administered opioid analgesics and local instillations of lidocaine to reduce pain. After 2 months the animal is fully recovered general condition is favorable Histopathological examination has proven ceruminous gland adenocarcinoma. Surgical ablation of the external ear canal if ear tumors is recommended
\end{abstract}

Keywords: ceruminous gland adenocarcinoma, dog, ear canal

\section{INTRODUCTION}

Ceruminous gland adenocarcinomas are locally aggressive and can invade nearby structures including the bones of the skull. These tumors also have the potential to metastasize to the nearby lymph nodes, salivary glands, or lungs. Adenomas are benign tumors that can grow and compress tissues, but do not usually invade tissues or spread to other areas. Rarely, other cancers can occur in the ear canals, including squamous cell carcinoma or carcinomas of unknown origin. Other benign tumors include inflammatory polyps, papillomas, and basal cell tumors. Ear canal tumors can appear as firm nodules or plaques in the ear canals and can be pink, white, or purplish in color. These tumors can be ulcerated and cause bleeding or discharge from the ears. They can also cause odor from the ear, pain or itchiness. Vestibular signs, such as tilting of the head, falling to the side, circling, or difficulty blinking, can also be seen with these tumors due to invasion of the tumor or associated infection. Diagnosis of these tumors requires a biopsy. The treatment of choice for ear canal tumors is surgical excision. Aggressive surgery is the treatment of choice for invasive or deep penetrating tumors. This surgery is often referred to as a total ear canal ablation (TECA). Total ear canal ablation (TECA) refers to the removal of the entire external ear canal from the medial aspect of the pinna to the osseous tympanic bulla.

\section{AIMS AND OBJECTIVES}

The aim of the research in this paper is to highlight the importance of otoscopic examination 
if chronic otitis does not respond to repeated treatment. Presentation in the dynamics of a unilateral chronic otitis dog had the following objectives: history, clinical examination completed by the otoscopic examination, fine needle biopsy, radiological examination, surgery, histopathological diagnosis

\section{MATERIALS AND METHODS}

For history shows that the animal has been treated regularly for 3 years initially with antibiotics and local anti-inflammatory and then about unilateral chronic otitis overview. Clinical manifestations gradually intensifies, the time between treatments decrease. Clinical examination shows local sensibility to palpation. We noticed a reddish color abundant secretion, auricular mucosa hyperemia. The exam was performed otoscopics difficult because of the narrowing of the ear canal. Deeply in the vertical portion of the duct auricular we observed a tumor duct obstruction. On radiographs, posterior to the tympanic membrane effusion was found without changes of adjacent bone structure. Initially we decided to perform lateral ear canal resection, but during surgery we observed that advanced tumors throughout the horizontal portion of the ear canal and tympanic membrane passing occupies part of the tympanic bulla. Therefore we decided a total ear canal ablation (TECA).

\section{RESULTS AND DISCUSSION}

Signs of ear infection or ear canal tumors include head shaking, scratching the ear, auricular congestion, crying in pain, sensitivity to touch the ear, ear canal narrowing, odor ear, auricular secretions reddish or yellow-green. In this case, the 5-year-old, female, German shorthaired Pointed, used to hunt, swim in the lake, shows unilateral otitis;Topical antibiotics and antiinflamatory medication, ear cleaning and ear flushing without properly treat otitis creates a vicious circle by accentuating mechanical irritation. Five days before surgery the animal received topical (ciprofloxacin- Ciplox) and systemic treatment with antibiotics(ceftriaxoneCefort and amoxicilinum+acid clavulanicum Amoxi-kel) and anti-inflammatory(tolfenamic acid- Tolfedine),. We used inhalatory anesthesia. Premedication with Acepromazine $50 \mu \mathrm{g} / \mathrm{Kg} \mathrm{im}$, 30 minutes before anesthesia and Butorphanol
$0.4 \mathrm{mg} / \mathrm{kg}$ im, 15 minutes before anesthesia. Induction with Propofol $4 \mathrm{mg} / \mathrm{kg}$ slow to effect, then intubation and Isoflurane maintenance $1 / \%$ - $3 \%$ in $100 \% 02$.

Auriculotemporal (cranial innervation) and great auricular nerve (caudolateral innervation) were blocked using Lidocaine $2 \%$ to contribute to intraoperative pain management, providing immediate pain relief, thus complement systemic analgesic modalities, referring to a multimodal analgesia. Initially we decided to perform lateral ear canal resection, but during surgery we observed that advanced tumors throughout the horizontal portion of the ear canal and tympanic membrane passing occupies part of the tympanic bulla. Therefore we decided a total ear canal ablation (TECA). When we opened the vertical portion of the ear canal we observed tumor occupying the entire horizontal portion we completely removed the external ear duct, then we examined the tympanic membrane. it was ruptured and friable tissue reddish color was present in the tympanic bubble. We deeply removed using scaler and we sutured anatomical structures with absorbable monofilament PDS 3/0.A wound soaker catheter was placed for administration of local anesthetic Lidocaine $2 \mathrm{mg} / \mathrm{kg}$ for post operative analgesia every 2 hours.Postoperatively, as for the most aural procedures NSAIDs alone are not expected to ensure sufficient analgesic, so we prescribed Carprofen $4 \mathrm{mg} / \mathrm{kg}$ initial dose combined with Tramadol $4 \mathrm{mg} / \mathrm{kg}$ every 8 hours and sistemic antibiotics.

\section{CONCLUSION}

Ceruminous gland adenocarcinomas are locally aggressive and can invade nearby structures including the bones of the skull. Total ear canal ablation (TECA) is the optimal treatment recommended.Superficial biopsies of these tumors are classified as polyps due to granulation tissue covering epithelium. Chronic recurrent otitis is necessary to be thorough investigated.

\section{REFERENCES}

1. Bischoff MG, Kneller SK(2004). Diagnostic imaging of the canine and feline ear. Vet Clin North Am Small Anim Pract 34:437-4580.

2. Rogers KS (1988). Tumors of the ear canal. Vet Clin North Am Small Animal Pract 18:859-868. 